\title{
Article
}

\section{Improvement of Preparation Method of UV-protecting Suspensions by the Use of Rheological Techniques}

\author{
Akio NASU $^{*}$ and Yasufumi OtsuBO ${ }^{* *}$ \\ *Material Science Research Center, Shiseido Co., Ltd. 2-2-1, Hayabuchi, Tsuzuki-ku, \\ Yokohama-shi, 224-8558 Japan \\ ${ }^{* *}$ Department of Urban Environment Systems, Graduate School of Engineering, Chiba University, \\ Yayoi-cho 1-33, Inage-ku, Chiba-shi, 263-8522 Japan
}

(Received : April 25, 2008)

\begin{abstract}
Fine particles of titanium dioxide $\left(\mathrm{TiO}_{2}\right)$ are effective UV-protecting inorganic materials in cosmetic products. The particles have the tendency to make flocs in liquids and the ability of UV light scattering of the suspensions strongly depends on the size of flocs. The degree of flocculation should be related to the rheological properties, and UVprotecting ability of the suspensions was investigated as a function of the size of flocs formed in a dispersion procedure during sample preparation. Essential requirements for UV-protecting cosmetics are strong cut-off ability for the UV light and high transparence for the visible light. Excellent balance of these optical properties was achieved in the suspensions of $\mathrm{TiO}_{2}$ particles with floc diameters ranging from 100 to $120 \mathrm{~nm}$. When the floc diameter was decreased less than $100 \mathrm{~nm}$ in the dispersion procedure, the suspensions turned to shear-thinning fluids from Newtonian ones. Such the change in rheological behavior can be used to obtain $\mathrm{TiO}_{2}$ suspensions possessing the designed UV-protecting ability and flow behavior.
\end{abstract}

Key Words: Titanium dioxide suspension / Silicone oil / Flocculation / Shear-thinning flow / UV protection

\section{INTRODUCTION}

Ultraviolet radiation (UV) from the sun in the wavelength range of 280-320 nm, which is referred to as UVB, is harmful to the human skin and excessive exposure causes sunburn, stain, and an increased risk for skin cancers. ${ }^{1-4)}$ Although several organic materials such as p-aminobenzoic acid and octyl salcylate are used as UV-protecting agents ${ }^{5,6)}$, ultrafine inorganic particles are very useful in sunscreen formulas owing to high safety and stability. The most widely used inorganic material is titanium dioxide $\left(\mathrm{TiO}_{2}\right){ }^{7.8)}$ The UVprotecting cosmetics are usually provided in the form of colloidal suspensions, because in practical use, they must be fluids or pastes to cover the skin. The UV protection is accomplished by light scattering, so the primarily important factors are the refractive index and diameter of particles. The fine particles such as $\mathrm{TiO}_{2}{ }^{9-11)}$ dispersed in a liquid can easily form flocs due to colloidal attractive forces. When the suspensions are flocculated to increase the floc diameter, the UV scattering or sun protection factor is decreased to a great

* To whom correspondence should be addressed: E-mail, akio.nasu@to.shiseido.co.jp extent and the appearance of skin becomes markedly white during application. In manufacturing process the selection of dispersion method is directly connected with the improvement of UV-protecting ability.

The most sensitive physical quantities which describe the flocculation levels of suspensions are the rheological values including steady-shear viscosity and dynamic viscoelasticity.

In previous papers ${ }^{12,13)}$, we have studied the relation between the rheology and UV-protecting ability for suspensions of ultra-fine particles of $\mathrm{TiO}_{2}$ and $\mathrm{ZnO}$ dispersed in a silicone oil in the presence of polyoxyethylene (POE)-modified silicones with different molecular structures. By the use of POEmodified silicone with linear conformation, in which the dimethylpolysiloxane (DMPS) moieties were incorporated on the ends of molecule as terminal groups (called ABA-type), we have succeeded in formulating the stable suspensions with low transmittance in the UV ranges. The high stability and low viscosity of suspensions produced by ABA-type dispersant can be attributed to the steric stabilization mechanism. The adsorbed conformation can be described through a model on block copolymers which contain adsorbing chains and stabilizing chains that dissolve in the solution. ${ }^{14-17)}$ The UV scattering ability of ultra-fine $\mathrm{TiO}_{2}$ particles was increased 
with the dispersion time. This indicates that there exists an optimum dispersion time for preparation of $\mathrm{TiO}_{2}$ suspensions with good optical properties. In the present paper, the research interests are focused on the effect of dispersion times. The relation between the rheological properties and UV-protecting ability will be discussed in connection with the changes in floc diameters during the dispersion processes.

\section{MATERIALS AND METHODS}

\subsection{Materials}

The suspensions were composed of ultra-fine $\mathrm{TiO}_{2}$ particles, dispersant, and silicone oil. The $\mathrm{TiO}_{2}$ (rutile) particles (MT-100TV from Tayca Co., Ltd.) had a spindle form with thickness of about $10 \mathrm{~nm}$ and length of 10-30 nm. The surfaces of $\mathrm{TiO}_{2}$ particles were treated with alumina and aluminum stearate, the content of which was $17 \%$ by weight. The density and surface area of surface-treated $\mathrm{TiO}_{2}$ were $2.7 \times 10^{3} \mathrm{kgm}^{-3}$ and $63.5 \mathrm{~m}^{2} \mathrm{~g}^{-1}$, respectively. The silicone oil used as the medium was decamethylcyclopentasiloxane(ShinEtsu Chemical Co., Ltd.), the density of which was $0.958 \times 10^{3} \mathrm{kgm}^{-3}$. Because of low molecular weight, the medium was volatile. The dispersant was a POE-modified silicone of ABA type polymer, whose molecular structure is shown in Fig. 1. The dispersant was synthesized from monoterminal reactive silicone oil (Silaplane from Chisso Co.), POE and hexamethylene diisocyanate. The molecular weights of POE moiety and total chain were 1,500 and 11,500, respectively, and HLB value was 2.6.

The ultra-fine $\mathrm{TiO}_{2}$ particles were dispersed by a paint shaker (Asada Co., Ltd.), in which the glass beads with a diameter of $1 \mathrm{~mm}$ were added at a filling factor of 0.5 by volume. The frequency of vibration was $11 \mathrm{~s}^{-1}$. The shaking time was changed in the range of 1 to $360 \mathrm{~min}$. The suspension at the particle concentration of $40 \%$ by weight was prepared for rheological measurements. The concentration of dispersant was $10 \%$ by weight based on the continuous medium. The amounts of adsorption of dispersant on the $\mathrm{TiO}_{2}$ particles are $1.6 \mathrm{mgm}^{-2}$ that corresponds to the concentration of about $3.6 \mathrm{wt} \%$ in $40 \mathrm{wt} \%$ suspensions. Therefore, the full coverage

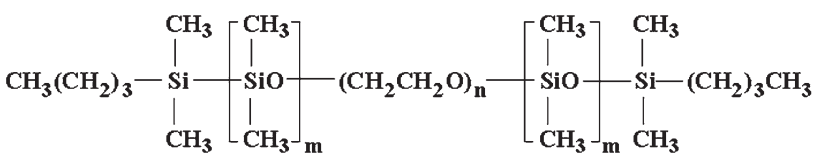

Fig. 1. Molecular structure of a dispersant. of particle surfaces with dispersant takes place in the sample suspensions.

\subsection{Methods}

Steady-flow and dynamic viscoelastic properties were measured with a cone-and-plate geometry on a stresscontrolled rheometer (AR-1000N manufactures by TA Instruments Co., Ltd.). The cone diameter was $40 \mathrm{~mm}$ and the gap angle between the cone and plate was $4^{\circ}$. The shear rates were from 0.1 to $500 \mathrm{~s}^{-1}$ in steady-flow measurements. The dynamic viscoelasticity was measured as a function of frequency at a small stress in the linear regions. The angular frequencies were from 0.02 to $10 \mathrm{~s}^{-1}$ and the measuring temperature was $25^{\circ} \mathrm{C}$.

Ultraviolet-visible light transmittances were measured by a spectrophotometer (U-3500 manufactured by Hitachi). The suspensions were diluted with decamethylcyclopentasiloxane and the $10 \mathrm{wt} \%$ suspensions were spread onto a piece of quartz plate $(50 \times 50 \times 5 \mathrm{~mm})$ to be $10 \mu \mathrm{m}$ in thickness by an applicator (Yahata Seiki Seisakusyo). The light transmittances were measured in the range of 280 to $700 \mathrm{~nm}$ wavelengths.

Floc diameters were measured by a particle size analyzer (Zetasizer Nano manufactured by Malvern), which was based on the dynamic light-scattering method. The $\mathrm{TiO}_{2}$ suspensions prepared for rheological measurements were diluted to $1.0 \mathrm{wt} \%$.

\section{RESULTS}

Figure 2 shows the average diameter of flocs plotted against the dispersion time for suspensions right after and 60 days

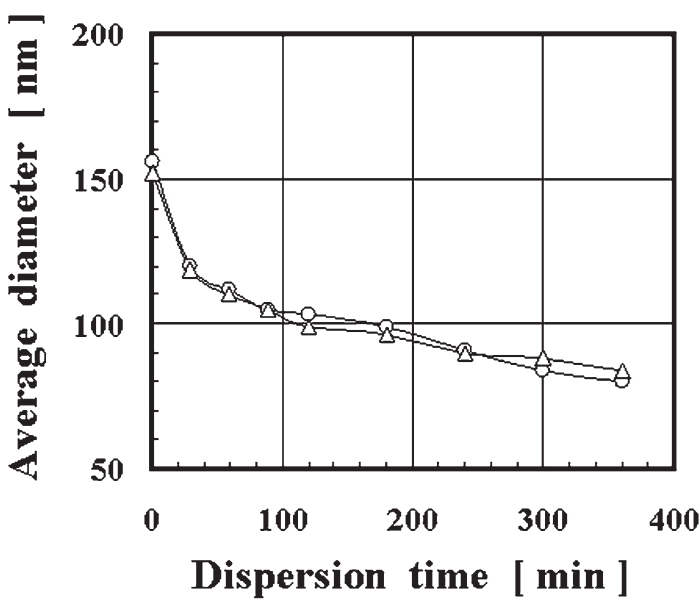

Fig. 2. Average diameter of flocs plotted against the dispersion time for suspensions right after preparation $(\bigcirc)$, and those with 60 -day aging after the preparation $(\triangle)$. 
after the preparation. The dispersion time is the shaking period applied to samples for preparation by a paint shaker. The floc diameter is not influenced by long-term storage, but determined by the dispersion time. After the rapid drop at the beginning, the floc diameter gradually decreases with increasing dispersion time. Before the sample preparation, the $\mathrm{TiO}_{2}$ particles are stored in ambient air conditions and are highly flocculated (often called "agglomerated" when fine dry particles make clusters in ambient air) by various types of forces such as capillary forces due to condensed water between particles and electrostatic static forces due to surface charges. At the first stage of preparation, the suspensions consist of a collection of large flocs, in which the air is entrapped as the dispersed phase. When subjected to high shear fields, the flocs are broken down by hydrodynamic forces. In addition, the exchange of entrapped air for the medium takes place and finally the air is released from the systems. It must be stressed that after the dispersion time of about $6 \mathrm{hr}$, the suspensions are not dispersed to primary particles.

Figure 3 shows the shear rate dependence of viscosities for suspensions right after the preparation obtained at different dispersion times. In short dispersion times, the flow behavior is nearly Newtonian with low viscosity. With increasing the dispersion time, the viscosity increases over the entire range of shear rates. The viscosity enhancement is larger at lower shear rates, resulting in the shear-thinning flow profile. If the suspensions are dispersed to non-interacting particles, the relative viscosity which is defined as a ratio of the viscosity of the suspension relative to that of the medium is estimated to be about 1.5 for 19.1 vol\% suspension, according to

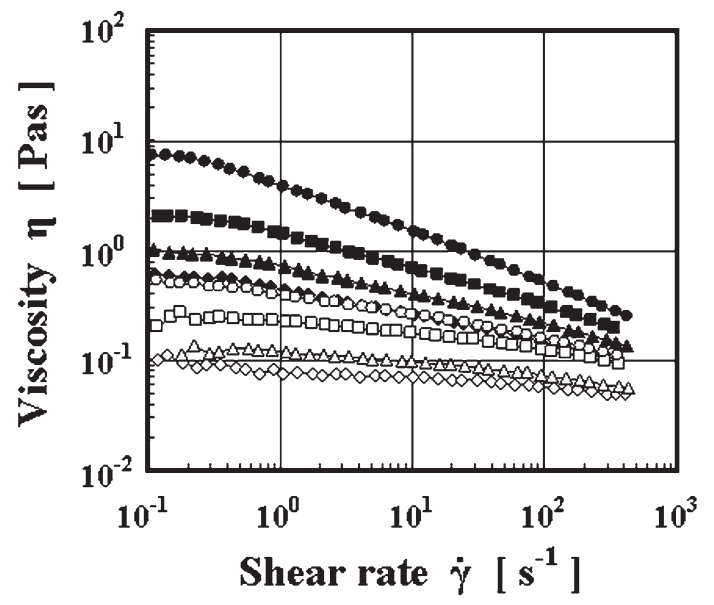

Fig. 3. Shear rate dependence of viscosities for suspensions right after preparation at different dispersion times: $(\diamond) 1 ;(\triangle)$ 30; $(\square)$ 60;

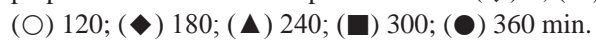

the Einstein's classical theory. The relative viscosity of suspensions at the shear rate of $10^{2} \mathrm{~s}^{-1}$ is in the range of 4.2-38, as the viscosity of medium is $1.3 \times 10^{-2}$ Pas. The high values of relative viscosity implies that the flocculation is induced in suspensions.

Figure 4 shows the shear rate dependence of viscosities for suspensions stored in a quiescent state for 60-day aging after the preparation at different dispersion times. Viscosities of suspensions sheared for more than $240 \mathrm{~min}$ in the dispersion process drastically increase, especially at low shear rates. The flow profiles become much more shearthinning. The shear-thinning flow of flocculated suspensions can be explained by the progressive breakdown of flocs by hydrodynamic forces.

The comparison of Figs. 3 and 4 suggests that the flocculation is significantly enhanced by aging. In the preparation process, the diameter of flocs decreases with increasing dispersion time, and they may act as primary flow units in suspensions. As mentioned above, these primary flow units are not broken down even in very violent shear fields applied in rheological measurements. Presumably the floc structure in suspensions may be described by a model in which the primary flocs are connected by weak inter-particle bonds. Without additives, it was impossible to prepare the suspensions of $\mathrm{TiO}_{2}$ particles in decamethylcyclopentasiloxane at a particle concentration of $40 \mathrm{wt} \%$. The POE-modified silicone is a necessary ingredient and act as a dispersant. The weak interactions may be generated between primary flocs.

For better understanding of flocculated structure, the dynamic viscoelasticity was measured for suspensions with 60-day aging. Figure 5 shows the frequency dependence

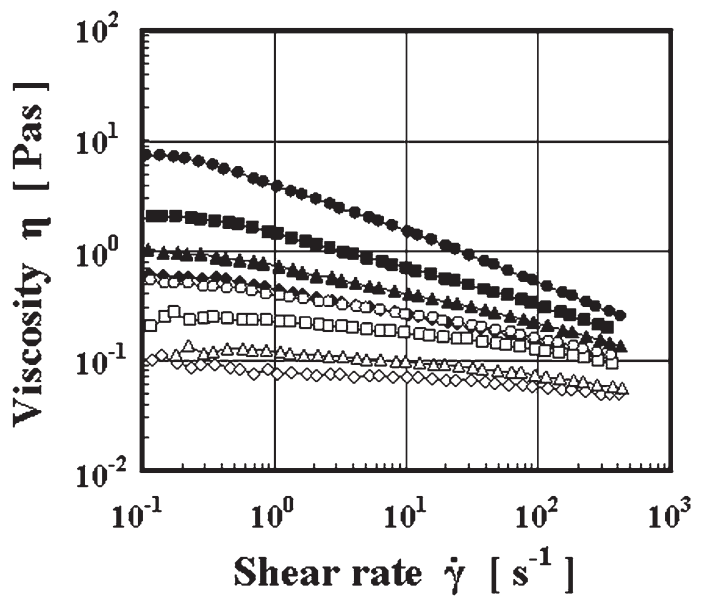

Fig. 4. Shear rate dependence of viscosities with 60-day aging after the preparation at different dispersion times: $(\diamond) 1 ;(\triangle)$ 30; $(\square)$ 60;

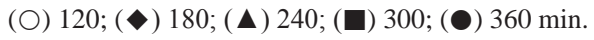


of storage modulus for suspensions prepared at different dispersion times. With increasing the dispersion time, the storage modulus at a given frequency rapidly increases as expected from the viscosity behavior. It is well known that the dynamic viscoelastic function of flocculated suspensions shows a plateau at low frequencies. The plateau may be the manifestation of the presence of network structure which is developed by rigid bonds. In Fig. 5, the storage modulus even for suspensions obtained at long dispersion times gradually decreases at low frequencies. The existence of terminal flow region in frequency-dependent curves implies that the bonds between particles relax by thermal energy. The suspensions can be regarded as viscoelastic liquids.

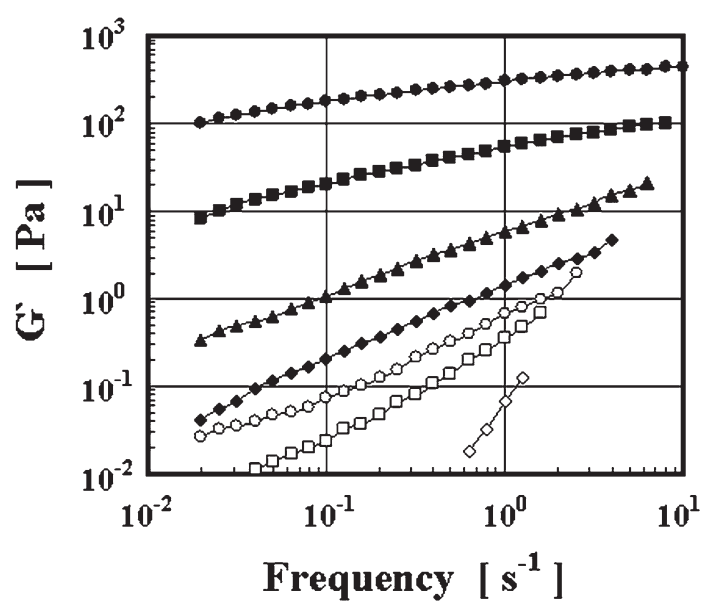

Fig. 5. Frequency dependence of storage modulus for suspensions with 60-day aging after the preparation at different dispersion times:

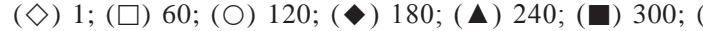
$360 \mathrm{~min}$.

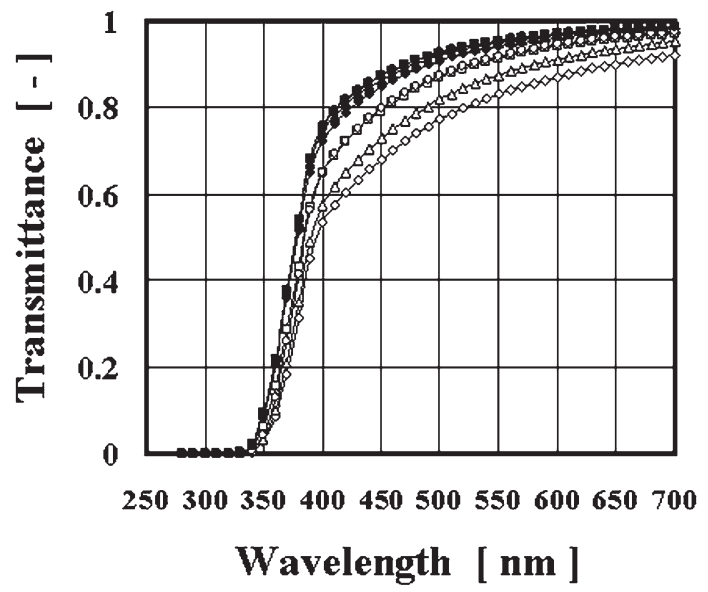

Fig. 6. Wavelength dependence of transmittance for suspensions with 60-day aging after the preparation at different dispersion times:

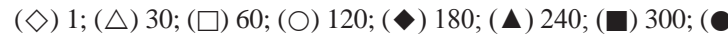
$360 \mathrm{~min}$.
Figure 6 shows the wavelength dependence of transmittance for suspensions with 60-day aging after the preparation at different dispersion times. The suspensions were diluted to $10 \mathrm{wt} \%$ prior to the optical measurements. At wavelengths below $350 \mathrm{~nm}$, the transmittance is very low. The suspensions show the excellent hiding power in the UV range. On the contrary, the suspensions show the high transmittance at long wavelengths. The transmittance is drastically changed in the wavelength range of 350-400 $\mathrm{nm}$. One can find that the increase of dispersion time causes the increase of transmittance in the whole range of wavelength.

\section{DISCUSSION}

The purpose of the present study is to understand the relation among the rheological properties, floc diameters, and UV-scattering ability for $\mathrm{TiO}_{2}$ suspensions, and to establish the preparation method of UV-protecting cosmetics with high performance. With respect to the optical properties of cosmetic products, the essential requirements are the good hiding power in the UV range and high transparency in the visible range. The suspensions with excellent UV-scattering ability are not necessarily accepted for cosmetic use, but the balance of transmittance in UV and visible regions is important.

Figure 7 shows the transmittance at 360 and $550 \mathrm{~nm}$ plotted against the floc diameter for suspensions stored in a quiescent state for 60 days. The transmittance in UV region is desired to be less than 0.2 and hence the floc diameter should be larger than $100 \mathrm{~nm}$. On the other hand, the transmittance in visible region is desired to be larger than 0.9. This corresponds to

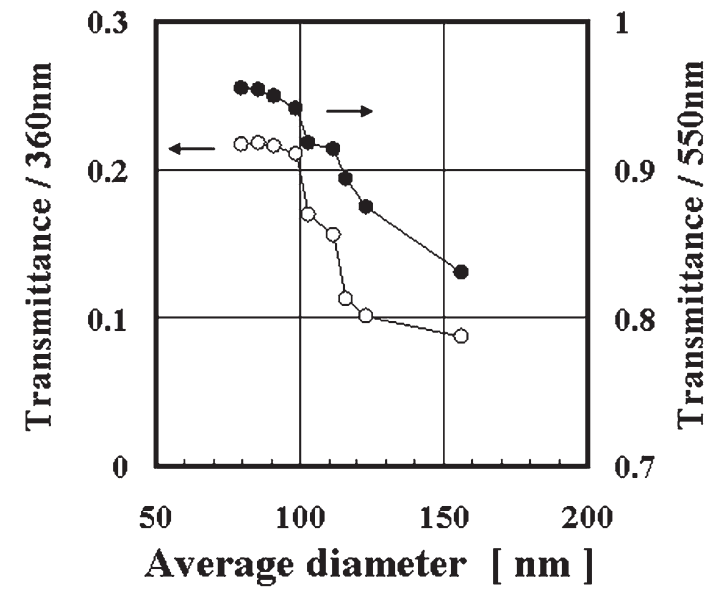

Fig. 7. Dependence of transmittance at wavelengths of 360 and $550 \mathrm{~nm}$ on the average floc diameter for suspensions with 60-day aging after the preparation. 
the suspensions which consist of flocs with diameters smaller than $120 \mathrm{~nm}$. Therefore, the good balance of optical properties is achieved for suspensions with floc diameters in the range of 100-120 nm. According to previous work, the optimum average diameter is in the range of $60 \sim 120 \mathrm{~nm}$ for the UVprotection by $\mathrm{TiO}_{2}$ particles.

Let us now consider the preparation procedures of suspensions which satisfy the good balance of optical properties. The ratio of viscosity at $1 \mathrm{~s}^{-1}$ relative at to $100 \mathrm{~s}^{-1}$ is employed as a measure to characterize the degree of shearthinning profile. Combining Figs. 2 and 3, the viscosity ratio is plotted against the floc diameter in Fig. 8 for suspensions right after preparation and those with 60-day aging after the preparation. During the 60-day aging, the shear-thinning tendency is enhanced for suspensions with small flocs, while the aging does not significantly influence the viscosity behavior for Newtonian suspensions which consist of large flocs. To keep the stable rheological properties in suspensions for a long period, the suspensions with the 60-day aging are more preferable. We can easily find from Figure 8 that the flow profile drastically changes at about $100 \mathrm{~nm}$. It should be noted that this changing point is comparable to the optimum floc diameters discussed above. Therefore, the rheological transition from Newtonian to shear-thinning profiles can be used as a controlling parameter in preparation of suspensions with good optical properties. The method of preparing the suspensions with good hiding power in the UV range and high transparency in the visible range can be established through the rheological techniques.

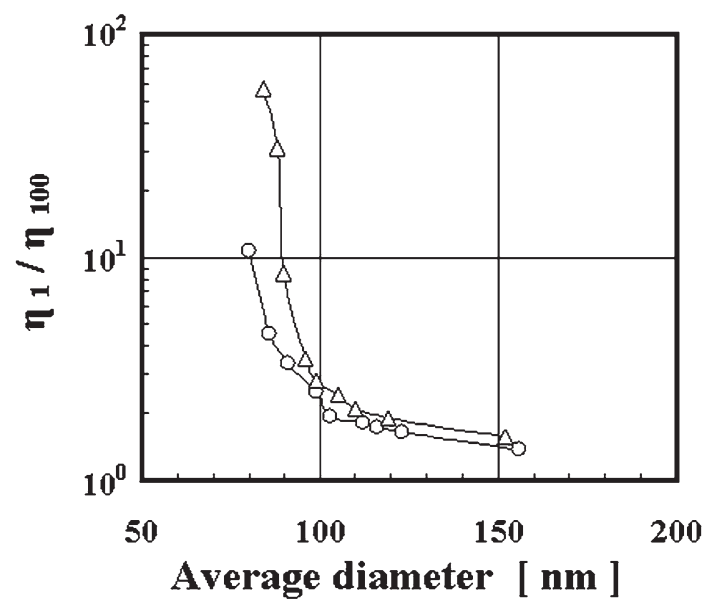

Fig. 8. Dependence of the viscosity ratio on the average floc diameter for suspensions right after preparation $(\bigcirc)$, and those with 60-day aging after the preparation $(\triangle)$.

\section{CONCLUSIONS}

The relations among the rheological properties, floc diameters, and UV-protecting ability have been examined for non-aqueous suspensions of $\mathrm{TiO}_{2}$ particles. The suspensions consisting of flocs with diameters in the range of 100-120 nm provide the good scattering ability in the UV range and high transparency in the visible range. With increasing the dispersion time, the floc diameter decreases and approaches about $100 \mathrm{~nm}$ the flow behavior changes from Newtonian behavior to shear-thinning one. The transition of flow profiles can be used as a controlling parameter in preparation of excellent UV-protecting suspensions.

\section{REFERENCES}

1) Kawagishi N, Hashimoto $Y$, Takahashi H, Ishida-Yamamoto A, Iizuka H, J Dermatol Sci, 18, 43 (1998).

2) Kollias N, Sayre RM, Zeise L, Chedekel MR, J Photochem Photobiol B: Biol, 9, 135 (1991).

3) Motoyoshi K, Ota Y, Takuma Y, Takenouchi M, Cosmet Toilet, 113, 51 (1998).

4) Lavker R, Kaidbey K, J Invest Dermatol, 108, 17 (1997).

5) Kligman LH, Akin FJ, Kligman AM, J Invest Dermatol, 78, 181 (1982).

6) Kligman LH, Akin FJ, Kligman AM, J Invest Dermatol, 81, 98 (1983).

7) Lansdown ABG, Taylor A, Int J Cosmetic Sci, 19, 167 (1997).

8) Bennat C, Müller-Goymann CC, J Cosmetic Sci, 22, 271 (1997).

9) Yang HG, Li CZ, Gu HC, Fang TN, J Colloid Interface Sci, 236, 96 (2001)

10) Doi M, Kawaguchi M, Kato T, Colloids Surf A, 211, 223 (2002).

11) Umeya K, Kanno T, J Rheol, 23, 123 (1979).

12) Nasu A, Otsubo Y, J Colloid Interface Sci, 296, 558 (2006).

13) Nasu A, Otsubo Y, J Colloid Interface Sci, 310, 617 (2007).

14) Milner ST, Witten TA, Cates ME, Macromolecules, 21, 2610 (1988).

15) Evers OA, Scheutjens JMHM, Fleer GJ, Macromolecules, 24, 5558 (1991).

16) Costello BA, Luckham PF, Th. F. Tadros ThF, J Colloid Interface Sci, 156, 72 (1993).

17) Wijmans CM, Leermakers FAM, Fleer GJ, J Colloid Interface Sci, 167, 124 (1994). 